\title{
Variability of diagnostic criteria and treatment of Idiopathic Nephrotic Syndrome across European countries
}

\author{
Georges Deschênes ${ }^{1}$, Marina Vivarelli ${ }^{2}$ \& Licia Peruzzi ${ }^{3}$,
}

On behalf of the ESPN Working Group on Idiopathic Nephrotic Syndrome*

1. Pediatric Nephrology Unit, Université Sorbonne Paris Cité, APHP Robert-Debré, 48 Bd Sérurier F-75019, Paris France georges.deschenes@aphp.fr

2. Division of Nephrology and Dialysis, Bambino Gesù Children's Hospital and Research Institute, Piazza Sant'Onofrio, 4, 00165 Rome, Italy marina.vivarelli@opbg.net

3. Nephrology Dialysis Transplantation Unit, Città della Salute e della Scienza, Regina Margherita Children's Hospital, Corso Bramante, 88 - 10126 Torino Turin, Italy licia.peruzzi@unito.it 


\section{* Working Group on Idiopathic Nephrotic Syndrome - list of Coauthors}

H. Alpay Marmara University Medical Faculty, Istanbul, Turkey harika.alpay@gmail.com

A. Alvaro Madrid Hospital Vall d'Hebron, Barcelona, Spain amadrid@vhebron.net

R. Andersen Aarhus University Hospital, Aarhus, Denmark rfa@clin.au.dk

M. Bald Olgahospital, Stuttgart, Germany m.bald@klinikum-stuttgart.de

E. Benetti Department of Woman's and Child's Health, AOP-University of Padova, Italy elisabene@libero.it

E. Berard Hôpitaux pédiatriques universitaires de Nice, Nice, France berard.e@chu-nice.fr

D. Bockenhauer Great Ormond Street Hospital, London, UK d.bockenhauer@ucl.ac.uk

O. Boyer Hôpital Necker, APHP, Université Paris-Descartes, Paris, France olivia.boyer@aphp.fr

D. Brackman Haukeland University Hospital, Bergen, Norway damien.marie.michel.brackman@helse-bergen.no

C. Dossier APHP Robert-Debré, Paris France claire.dossier@aphp.fr

Z. Ekinci Kocaeli Çocuk Nefroloji ve Romatoloji Bilim Dalı, Umuttepe, Türkiye zekinci@outlook.com

F. Emma Bambino Gesù Children's Hospital and Research Institute, Roma, Italy francesco.emma@opbg.net

B. Enneman KU Leuven and University Hospitals Leuven, Leuven, Belgium, benedicte.eneman@uzleuven.be

L. Espinosa-Roman Paediatric Nephrology. Hospital Universitario La Paz. Madrid. Spain.lespinosar@salud.madrid.org

M. Fila Hôpital Arnaud de Villeneuve, Montpellier, France m-fila@chu-montpellier.fr

L. Ghio U.O.C. Nefrologia e Dialisi Pediatrica - Fondazione IRCCS Cà Granda Milano, Italy luciana.ghio@policlinico.mi.it

J. W. Groothoff Emma Children's Hospital, Amsterdam, The Netherlands j.w.groothoff@amc.uva.nl

V. Guigonis Limoges University Hospital, Limoges, France v.guigonis@gmail.com

A. Jankauskiene Pediatric Center, Vilnius University, Vilnius, Lithuania augustina.jankauskiene@mf.vu.It

M. Kagan, Orenburg regional children's hospital, Orenburg, Russia mkaganorenburg@yahoo.com

M. Kovacevic, Institute for Child and Youth Health Care in Novi Sad, Serbia dr.maja.kovacevic@gmail.com

M.J. Kemper University Medical Center Hamburg-Eppendorf, Hamburg, Germany kemper@uke.de

E. Levtchenko KU Leuven and University Hospitals Leuven, Leuven, Belgium elena.levtchenko@uzleuven.be

S. Maringhini Ospedale dei Bambini "G. Di Cristina" A.R.N.A.S. Palermo, Italy s.maringhini@ospedalecivicopa.org

S. Mir Ege University Faculty of Medicine, İzmir,Turkey sevgi.mir@ege.edu.tr

A. Mitsioni P. \& A. Kyriakou Children's Hospital, Athens, Greece amitsioni@icloud.com

M. Mizerska-Wasiak and K. Wasiak, Medical University of Warsaw, Warsaw, Poland wasiaczki@wp.pl

A. Moczulska Jagiellonian University Medical College, Cracow, Poland amoczulska@cm-uj.krakow.pl

G. Montini University of Milan, Ospedale Maggiore Policlinico, Milano, Italy giovanni.montini@unimi.it

L. Murer Department of Woman's and Child's Health, AOP-University of Padova, Italy. luisa.murer@sanita.padova.it

M. Nuutinen Department of Pediatrics, University of Oulu, Oulu, Finland mnuutine@cc.oulu.fi

V. Obukhova Research Institute of Pediatrics and Children Surgery, Moscow, Russia obuhova.v@mail.ru

J. Oh University Medical Center Hamburg-Eppendorf, Hamburg, Germany j.oh@uke.de

O. Ozkaya Fatih Sultan Mehmet Training and Research Hospital, Istanbul, Turkey ozanozkaya@yahoo.com

T. Papalia Azienda Ospedaliera, Italy, Cosenza teresapapalia@alice.it

A. Peco Antic BEL MEDIC General Hospital, Belgrade, Serbia amira@udk.bg.ac.rs

C. Pecoraro Nephrology and Dialysis, Children Hospital Santobono-Pausilipon, Naples Italy pecoraro@unina.it

A. Pena-Carrion Hospital Universitario La Paz. Madrid, España antonia.pena@salud.madrid.org

E. Petrossian Russian National Research Medical University, Moscow, Russia ed3565@ya.ru

C. Pietrement, Centre Hospitalier Universitaire de Reims, France cpietrement@chu-reims.fr

L. Prikhodina Pirogov Russian National Research Medical University, Moscow, Russia Iprikhodina@yahoo.com

U. Querfeld Charité Universitätsmedizin, Berlin, Germany uwe.querfeld@charite.de

S. Rittig Aarhus University Hospital, Aarhus, Denmark rittig@clin.au.dk

M. Saleem, School of Clinical Sciences, University of Bristol, UK $\underline{\text { m.saleem@bristol.ac.uk }}$

M. Saraga School of Medicine, University of Split, Split, Croatia msaraga@kbsplit.hr

N. Savenkova Saint-Petersburg State Pediatric Medical University, St Petersburg, Russia savenkova.n.spb@mail.ru

L. Sever İstanbul University Cerrahpaşa Faculty of Medicine, İstanbul, Turkey severlale@hotmail.com

K. Tullus Great Ormond Street Hospital, London, UK Kjell.Tullus@gosh.nhs.uk

T. Ulinski, Armand-Trousseau Hospital, Paris, France tim.ulinski@aphp.fr

J. Vande Walle, Safepedrug, University Hospital Gent, Gent, Belgium Johan.VandeWalle@uzgent.be

J. Vara Martin Hospital Universitario 12 de Octubre, Madrid, España julia.vara@salud.madrid.org

N. Webb University of Manchester, Manchester Academic Health Science Centre, Royal Manchester Children's Hospital, Manchester, UK Nicholas.Webb@cmft.nhs.uk

L.T. Weber University Hospital of Cologne, Cologne, Germany lutz.weber@uk-koeln.de

A. Zurowska Medical University Gdansk, Poland azur@gumed.edu.pl 


\section{Contribution of authors}

Georges Deschênes and Licia Peruzzi conceived the questionnaires and wrote the paper

Marina Vivarelli wrote the paper with LP and GD

Coauthors of the ESPN working group on Idiopathic nephrotic Syndrome answered to the surveys

Among coauthors, Olivia Boyer, Detlef Bockenhauer, Etienne Berard, Lutz Weber \& Lale Sever kindly reviewed the paper. 


\section{What is known}

Steroids and Immunosuppressants are the universal treatment of Idiopathic Nephrotic Syndrome

\section{What is new}

The variability of treatments and strategy of treatment in European centers of pediatric nephrology 


\section{Abstract}

The aim of the surveys conducted by the Idiopathic Nephrotic Syndrome Working Group (WG) of the ESPN was to study the possible variability of treatment in Europe at different stages of the disease by means of questionnaires sent to members of the working group. Four surveys have been completed: the treatment of the first flare, the treatment of the first relapse and the issue of steroid dependency, the use of rituximab, the management of steroid resistant patients. A uniform treatment of the first flare was applied in only 3 countries and 10 additional centers have adopted one of the 3 main protocols. Reported treatment of the first relapse was relatively uniform whereas the use of additional immunosuppressant in steroid dependency was widely variable. Rituximab had already been used in hundreds of patients although the formal evidence of efficiency in steroid dependency was relatively recent at the time of the survey. The definition of steroid resistance was variable in the European centers but strikingly, the first line treatment was uniform throughout the centers and included the combination of prednisone plus calcineurin antagonists.

Conclusion. The variability in the approach of Idiopathic Nephrotic Syndrome is unexpectedly large and affects the treatment of the first flare, the strategies in case of steroid dependency as well as the definitions of steroid resistance. 


\section{List of Abbreviations}

$\begin{array}{ll}\text { APN } & \text { Arbeitgemeinshaft für Pediatrische Nephrologie } \\ \text { ESPN } & \text { European Society of Pediatric Nephrology } \\ \text { GNP } & \text { German Society for Pediatric Nephrology } \\ \text { KDIGO } & \text { Kidney Disease Improving Global Outcomes } \\ \text { INS } & \text { Idiopathic Nephrotic Syndrome } \\ \text { ISKDC } & \text { International study of Kidney Disease in Childhood } \\ \text { IVMP } & \text { Intravenous methylprednisolone } \\ \text { MP } & \text { Methylprednisolone } \\ \text { SNP } & \text { Société de Néphrologie Pédiatrique } \\ \text { WG } & \text { Working Group }\end{array}$


Idiopathic nephrotic syndrome (INS) is not a frequent disease but not an orphan disease. The annual incidence in the population below 16 years of age is between 1.2 to 3.4 new cases per 100,000 [6]. It means that 1000 to 1500 new cases are occurring yearly in the European Union. Roughly half of them will be steroid dependent and will have a median course of 10 years, meaning that 6000 to 16,000 pediatric patients (equivalent to 6 to 18 per 100.000 of general population < 16 years of age) are currently treated with steroids or immunosuppressive drugs. One of the main difficulties in gathering multicentre data for clinical studies on INS is due to the adoption of different treatment protocols as well as the definition of outcomes and resistance to treatment. This issue is particularly important in INS where elements of the diagnosis and the prognosis are based on the response to the very initial phase of the treatment.

The aim of the surveys conducted by the Idiopathic Nephrotic Syndrome Working Group (WG) of the ESPN was to study the possible variability of treatment in Europe at different stages of the disease by means of questionnaires sent to members of the WG. These questionnaires aimed at exploring different debated issues of INS treatment. The global conclusions are that the variability is unexpectedly large and not always understandable, even when a general consensus had apparently been reached.

\section{Methods}

Four surveys have been completed from 2013 to 2015 and sent to the 52 members of the INS working group.

Survey 1 (first flare). The treatment of the first flare was investigated using a template where the classical protocols were recalled along with a space for the single center's individual approach. Each participant was asked to fill the available cells of the column space entitled "your protocol" with the local protocol of treatment of the first flare. 
Survey 2 (First relapse and steroid dependency). The treatment of the first relapse and the management of steroid dependent patients were investigated using a clinical case. The first query aimed at describe the treatment of the first relapse in each center. According to the answer, a second query was on the timing and choice of additional immunosuppressive treatment in each center.

Survey 3 (Rituximab). The use of rituximab was investigated using two successive questionnaires. The first questionnaire mainly aimed at define the criteria to treat with Rituximab (steroid dependency, duration or severe complication of the disease) and the second questionnaire was dedicated to the technical points: dose, number of infusions for the initial cure, duration of $\mathrm{B}$ cell depletion, antimicrobial prophylaxis, withdrawal of conventional immunosuppressive drugs and steroids.

Survey 4 (Steroid resistant forms). The management of steroid resistant patients was investigated using a template to fill in. The template contained open criteria of the definition, the use of renal biopsy and the access to genetic testing first line treatment, duration of the treatment prior to consider failure, criteria of success.

\section{Results}

The surveys were sent to 52 centers and answered by 40 centers from 19 countries. There were 29, 16, 27 and 18 answers for Survey 1, 2, 3 and 4 respectively (Table I).

First flare (Table II). The questionnaire was returned by 26 individual centers. In addition, 3 countries, Denmark, Germany and France that applied a uniform nationwide treatment were considered as one answer while they represent dozens of individual centers. Surprisingly, those 3 protocols were relatively similar but differed by the cumulative dose of prednisone (3360 mg/m² for Denmark and Germany and $3390 \mathrm{mg} / \mathrm{m}^{2}$ for France), the use of intravenous methylprednisolone in case of oral steroid resistance (Germany and France), and the tapering 
sequence (only in France). In other centers, the variability of the cumulative dose ranged from $2240 \mathrm{mg} / \mathrm{m}^{2}$ to $4245 \mathrm{mg} / \mathrm{m}^{2}$. Consistently the duration of treatment also varied from 8 to 24 weeks. Other important results showed that 1) all centers gave $60 \mathrm{mg} / \mathrm{m}^{2} /$ day of prednisone (n $=14)$ or prednisolone $(n=15)$ to start the treatment; 2$)$ all centers were limiting the daily dose during the first weeks of treatment, 18 of them to less than $60 \mathrm{mg} /$ day meaning that patients over $1 \mathrm{~m}^{2}$ (about $30 \mathrm{Kg}$ body weight) were receiving less than the nominal cumulative dose mentioned in Table I; 3) 20/29 protocols included a sequence of several steps of steroid tapering before withdrawal; 4) 20/29 were using an intravenous (IV) methylprednisolone test in case of steroid resistance at the end of the period of full oral daily dose. Another unexpected fact was the variability of the protocols within large countries (Italy, Russia, Spain and Turkey) as well as within smaller countries (Belgium, Croatia and Serbia). Finally, half of the centers (13/26) were following one of the 3 main protocols emerging from the literature (Table IV): 2 centers (\#01 to \#02, Table I) had a protocol close to the model of the International Study of Kidney Diseases in Children (ISKDC, Table IV), 7 centers (\#13 to \#19, Table I) had a protocol close to the model of the German Society for Pediatric Nephrology (GPN, Table IV) and 4 (\#24 to \#27, Table I) had a protocol close to that of the French Société de Néphrologie Pédiatrique (SNP, Table IV).

First relapse and steroid dependency. The survey adopted the form of a clinical quiz that was compiled by 16 centers. The clinical case presented a typical steroid dependent patient relapsing 5 days after the withdrawal of prednisone. Reported treatment of the first relapse was relatively uniform and consisted of a short period of daily prednisone limited to the time to obtain a remission in 1 center, plus 3 days in 9/16 centers and plus 5-7 days in 5 centers. Only one center reported treating the $1^{\text {st }}$ relapse similarly to a first flare in the absence of significant steroid toxicity. A single treatment with prednisone lasting from 1 to 6 months without any prevention of further relapses was the choice of 7 centers. A systematic 
prevention of further relapses was undertaken as soon as the first early relapse by 8 centers: 5 gave long lasting alternate day prednisone therapy, 1 levamisole, 1 cyclosporine and 1 an unconventional combination of levamisole, mycophenolate and subcutaneous polyclonal immunoglobulins. In the last center, a treatment with mycophenolate was given only in case of significant signs of steroid toxicity. Three centers also mentioned that cyclophosphamide or rituximab could be an option as soon as the first relapse in case of relapse prior to the withdrawal of steroids.

At the second relapse, immunosuppressant drugs were systematically given to patients in 9 centers. Mycophenolate was the first option in the majority of those centers and only 2 considered cyclophosphamide or rituximab at this stage. Four centers did not consider any systematic prevention of further relapses at this stage of the disease but mycophenolate or levamisole were conditioned to steroid toxicity in $3 / 4$ centers and cyclophosphamide in 1/4. The 3 last centers reported no answer for the second relapse and beyond.

Rituximab. The questionnaire was sent in 2 parts. Twenty-seven centers answered the first part and 13 the second part. One center had no experience at all. By contrast, several hundreds of patients have been treated with rituximab in the 26 other centers. Three centers reported more than 50 patients each and 4 other centers reported 20 to 50 patients each. The last 18 centers had a more marginal experience with less than 10 patients. The major indication of treatment was steroid dependency. Other frequent indications included steroid resistance (8 centers) and long lasting treatment with immunosuppressant (8 centers). A minimum of at least a 3 year-duration of nephrotic syndrome was reported by 7 centers but 2 centers had clearly no lower limit for prescription. Rituximab was the last line of treatment for 3 centers. Either non-compliance or complications were an indication in 6 centers. Strikingly, costs of rituximab were not covered by healthcare insurance in Belgium and Poland in 2015. 
Of the 13 centers that answered the $2^{\text {nd }}$ part of the questionnaire, 12 were initiating the treatment by 1 ( 8 centers) or 2 infusions at 1 week of distance ( 4 centers) while only 1 center followed the classic protocol consisting of 4 infusions in 4 weeks. The dose of one infusion was $375 \mathrm{mg} / \mathrm{m}^{2}$ for all centers except 1 that reported a unitary dose of $750 \mathrm{mg} / \mathrm{m}^{2}$. Reinfusion was performed if B cell repletion was observed within 6 months after the first infusion in 6 centers and only in case of relapse in 4 centers. A systematic long-lasting B cell depletion of 12 and 18 months was achieved in 2 centers. An alternate protocol adopted by 1 center was to treat any relapses with rituximab in steroid dependent patients, allowing to rapidly stop the oral treatment. A reinfusion was never done in one center. A withdrawal of all oral drugs was attempted by all centers with a delay varying from 1 month (in 3 centers) to 12 months.

Steroid resistant forms. Eighteen centers answered the survey. The heterogeneity of the definition of steroid resistance is shown in Table III. The first line treatment that was adopted by the 18 centers was the association of calcineurin inhibitors, either cyclosporine ( 15 centers) or tacrolimus ( 3 centers). The patient was considered multidrug resistant in case of no response to the association of calcineurin inhibitor and prednisone for a period of 3 months (2 centers), 6 months ( 8 centers) or 9 months ( 5 centers) ( 3 centers did not answer the question). Partial response to treatment was considered $50 \%$ proteinuria reduction by most centers and combined to serum albumin increase in 3 centers. In case of partial response, mycophenolate was added as a second line treatment in 13 centers. Only isolated centers propose as third line treatment with apheretic techniques, intravenous high doses immunoglobulin and rituximab. One center had no additional treatment in case of multidrug resistance. All centers decided to withdraw immunosuppressive therapies, shifting to exclusive supportive cares basing on unequivocal genetic results (all centers) or the development of renal failure (all centers).

\section{Discussion}


Idiopathic Nephrotic Syndrome affects children all over the globe and one should expect that they should be treated with common and shared protocols. Here, we report the results of surveys that concern the treatment of children with INS in the European area. The data are coming from a sample of the European centers of Pediatric Nephrology belonging to the INS working group of the ESPN and that answered to the 4 surveys. Despite a relatively low number of participating centers compared to the total number of centers in Europe that is clearly a limitation of the study, the surveys show large variations of treatment strategies at all steps of the management of idiopathic nephrotic syndrome in the European area as already reported elsewhere in the world [16,21].

Numerous guidelines, meta-analyses [9] and follow-up series on the treatment of the first flare of nephrotic syndrome have been released in the literature by Pediatric Nephrology societies, consortium of centers or single centers $[8,12,18,25]$. At least 7 randomized prospective trials have tested different protocols of steroid therapy [8]. The fact is that the literature shows substantial differences of treatment of the first flare according to countries and centers. In addition to the protocol described in the literature as the International Study of Kidney Disease in Childhood [3], the GPN (formerly named Arbeitgemeinshaft für Pädiatrische Nephrologie - APN) protocol [5] and the protocol of the Société de Néphrologie Pédiatrique [2], US and India guidelines recommend a protocol that is very similar to those of the GPN $[1,8]$. The protocol recommended by the KDIGO is less well defined in term of duration of treatment and composes with different sources [14]. The main details of protocols that are used in Europe are shown in Table IV. They are quite different in terms of duration and cumulated dose of steroid therapy, the tapering protocol and the option of intravenous methylprednisolone in case of oral steroid resistance. As a matter of fact, out of the European centers of Pediatric Nephrology, one half of the centers reported to use the ISKDC or the APN or the SNP protocols while the other half of centers have a local protocol that is more or 
less a mix of the 3 main protocols. The variability in dose and duration of prednisone (or prednisolone) has already been reported in Italy, not only in General Pediatrics Units but also in those specialized in Pediatric Nephrology [20]. Noteworthy, 2 centers using the classical ISKDC protocol and 3 using the APN protocol have reported the use of IV methylprednisolone in case of oral steroid resistance while this option is not classically included in those protocols.. The most surprising finding is that, according to this survey, a total of 13 European centers are treating patients below the KDIGO recommendations of minimum steroid therapy: less than 12 weeks of duration or less than $3360 \mathrm{mg} / \mathrm{m}^{2}$ of cumulative dose corresponding to the sum of 4 weeks at $60 \mathrm{mg} / \mathrm{m}^{2} /$ day and 6 weeks at 40 $\mathrm{mg} / \mathrm{m}^{2}$ e.o.d. [14]. Nevertheless, 2 recent prospective randomized trials have shown that a cumulative dose of $2240 \mathrm{mg} / \mathrm{m}^{2}$ of prednisone had the same effect on the outcome as 3500 and $3900 \mathrm{mg} / \mathrm{m}^{2}[24,26]$. By contrast, a less recent prospective trial from Japan also showed that patients under 4 years of age had a strong benefit of a high cumulative dose of prednisolone [11]. At the other end, none of the centers participating to this survey reported steroid overtreatment contrasting with a recent Italian report where patients in 2 pediatric nephrology units are treated with cumulative dose of prednisone over $5000 \mathrm{mg} / \mathrm{m}^{2}$ [20]. However, our data clearly highlights the need to discuss common and shared European guidelines aimed at optimizing steroid therapy according to one protocol with different options. Based on the data of the literature and on the experience of centers, European guidelines should at least state about uniform dose of steroids and define one option for the tapering protocol and the test with IV methylprednisolone.

In contrast with the treatment of the first flare, the steroid therapy of the first relapse which has never been the objective of a randomized prospective trial is very homogenous in Europe. All but one centers treat the first relapse with a shortened duration of daily prednisone and a rapid tapering of the dose from 0 to 7 days after the remission of proteinuria, consistent with 
the KDIGO recommendations [14]. At this point, differences between centers concern the duration of steroid therapy and the use of an additional second-line treatment. A prevention of relapses by either long lasting steroid therapy or levamisole was reported by seven centers as soon as the first relapse. Beyond the first relapse, KDIGO recommendations are vague, particularly on the chronology of each possible steroid sparing treatment. No difference are suggested between the treatments that prevent relapses as long as they are continued but have no remnant effect after withdrawal (levamisole, mycophenolate and calcineurin inhibitors) and those that clearly show the ability to disrupt the course of the disease in long term followup series (cyclophosphamide, rituximab) [14]. At the second relapse, nine European centers considered an additional treatment and four reported using an additional treatment only in case of steroid toxicity. Among the nine centers that reported an additional treatment, only two reported the early use of cyclophosphamide or rituximab. The duration of the disease was never explicitly mentioned as an indication of additional treatment.

Rituximab has now been fully recognized as an efficient treatment of steroid dependent patients with a high rate of evidence since the end of 2014 and the release of the first randomized controlled trial [13]. Nevertheless, the efficiency of rituximab has been empirically recognized by clinicians for several years [4] while more than 300 patients have been reported in the survey at the end of 2014. Variations in the use of rituximab are somehow limited: most of the centers reported the same unitary dose of $375 \mathrm{mg} / \mathrm{m}^{2}$ and the number of initial infusion was 1 or 2. Long-lasting B cell depletion [22] has been used by 2 centers but one should recall that the risk of life-threatening infections might be proportional to the duration of B cell depletion [23]. An alternative way might be to systematically treat relapses in steroid dependent patients with one infusion of rituximab in order to stop oral treatments rapidly and to delay the next relapse. Nevertheless, the best way to use rituximab remains to be defined in the near future. 
Steroid resistance is a condition of high morbidity leading to end stage renal failure or to long-lasting and heavy immunosuppressive drug therapy [17,27]. The definition of steroid resistance is very heterogeneous in the survey. Whereas the prevalence of steroid sensitivity is proportional to the duration and the cumulative dose of steroids [19], the resistance after 4 weeks of oral prednisone might have a different significance in term of difficulty to treat than the resistance after 8 weeks of oral prednisone plus 3 intravenous methylprednisolone pulses. Nevertheless, the first-line treatment is similar in all centers regardless to the definition. All centers reported using a first-line of prednisone and calcineurin antagonists, consistently with the KDIGO recommendations [15]. Mycophenolate is a common drug included in the maintenance treatment, specially in partial response. Positive genetic testing is a major cause of immunosuppressive treatment withdrawal in all centers suggesting that the first line treatment is systematically started before genetic results likely to be delayed in most cases. The other criteria that leads to withdrawal of immunosuppressive therapy is the time to unresponsiveness that ranges between 3 and 9 months, defining the multidrug resistance. In this case, experimental therapeutical options using plasma exchange or immunoglobulin removal are a choice for only 2 centers.

To conclude, a lot of work remains to be done in order to homogenize the treatment of idiopathic nephrotic syndrome based on high-quality evidence. The choice of treatment remains largely dependant on each physician's clinical experience at all steps. This variability of therapeutic approaches deserves some attention and supports the commitment of the INS Working Group of the ESPN to build guidelines and consensus. 


\section{Compliance with Ethical Standards}

Funding

This study has been supported by a grant of $1000 €$ from the European Society of Pediatric Nephrology

\section{Conflicts of interest}

Georges Deschênes declares that he has no conflict of interest and no financial relationship with the ESPN

Marina Vivarelli declares that she has no conflict of interest and no financial relationship with the ESPN

Licia Perruzi declares that she has no conflict of interest and no financial relationship with the ESPN

The list of coauthors declare no conflict of interest and no financial relationship with the ESPN

Ethical approval

All procedures performed in studies involving human participants were in accordance with the ethical standards of the institutional and/or national research committee and with the 1964 Helsinki declaration and its later amendments or comparable ethical standards.

It is clear that this paper obviously reports the routine clinical experience of medical teams on the treatment of human patients affected of a human disease but this article does not contain any experimental studies with human participants or animals performed by any of the authors. It is also clear that several thousand human patients are the core of this clinical experience but this paper does not report any specific information contained in patient files. Consequently, informed consent has not been obtained from individual participants. 


\section{References}

1. Bagga A, Ali U, Banerjee S, Kanitkar M, Phadke KD, Senguttuvan P, Sethi S, Shah M (2008) Management of steroid sensitive nephrotic syndrome: revised guidelines. Indian pediatrics 45:203-214. doi:

2. Berard E, Broyer M, Dehennault M, Dumas R, Eckart P, Fischbach M, Loirat C, Martinat L (2005) [Corticosensitive nephrotic syndrome (or nephrosis) in children. Therapeutic guideline proposed by the Pediatric Society of Nephrology]. Nephrologie \& therapeutique 1:150-156. doi: 10.1016/j.nephro.2005.06.003

3. Brodehl J, Ehrich JHH (1988) Short versus standard prednisone therapy for initial treatment of idiopathic nephrotic syndrome in children. Arbeitsgemeinschaft fur Padiatrische Nephrologie. Lancet 1:380-383. doi:

4. Cara-Fuentes G, Kairalla JA, Ishimoto T, Rivard C, Johnson RJ, Garin EH (2014) Rituximab in idiopathic nephrotic syndrome: does it make sense? Pediatr Nephrol 29:1313-1319. doi: 10.1007/s00467-013-2534-4

5. Ehrich JH, Brodehl J (1993) Long versus standard prednisone therapy for initial treatment of idiopathic nephrotic syndrome in children. Arbeitsgemeinschaft fur Padiatrische Nephrologie. European journal of pediatrics 152:357-361. doi:

6. El Bakkali L, Rodrigues Pereira R, Kuik DJ, Ket JC, van Wijk JA (2011) Nephrotic syndrome in The Netherlands: a population-based cohort study and a review of the literature. Pediatr Nephrol 26:1241-1246. doi: 10.1007/s00467-011-1851-8

7. Gellermann J, Ehrich JH, Querfeld U (2012) Sequential maintenance therapy with cyclosporin A and mycophenolate mofetil for sustained remission of childhood steroidresistant nephrotic syndrome. Nephrology, dialysis, transplantation : official publication of the European Dialysis and Transplant Association - European Renal Association 27:1970-1978. doi: 10.1093/ndt/gfr572

8. Gipson DS, Massengill SF, Yao L, Nagaraj S, Smoyer WE, Mahan JD, Wigfall D, Miles P, Powell L, Lin JJ, Trachtman H, Greenbaum LA (2009) Management of childhood onset nephrotic syndrome. Pediatrics 124:747-757. doi: 10.1542/peds.2008-1559

9. Hahn D, Hodson EM, Willis NS, Craig JC (2015) Corticosteroid therapy for nephrotic syndrome in children. The Cochrane database of systematic reviews:CD001533. doi: 10.1002/14651858.CD001533.pub5

10. Hamasaki Y, Yoshikawa N, Nakazato H, Sasaki S, Iijima K, Nakanishi K, Matsuyama T, Ishikura K, Ito S, Kaneko T, Honda M (2013) Prospective 5-year follow-up of cyclosporine treatment in children with steroid-resistant nephrosis. Pediatr Nephrol 28:765-771. doi: 10.1007/s00467-012-2393-4

11. Hiraoka M, Tsukahara H, Matsubara K, Tsurusawa M, Takeda N, Haruki S, Hayashi S, Ohta K, Momoi T, Ohshima Y, Suganuma N, Mayumi M (2003) A randomized study of two long-course prednisolone regimens for nephrotic syndrome in children. American journal of kidney diseases : the official journal of the National Kidney Foundation 41:1155-1162. doi:

12. Hodson EM, Willis, N.S., Craig J.C. (2008) Corticosteroid therapy for nephrotic syndrome in childhood (Review). The Cochrane Librairy doi:

13. Iijima K, Sako M, Nozu K, Mori R, Tuchida N, Kamei K, Miura K, Aya K, Nakanishi K, Ohtomo Y, Takahashi S, Tanaka R, Kaito H, Nakamura H, Ishikura K, Ito S, Ohashi Y (2014) Rituximab for childhood-onset, complicated, frequently relapsing nephrotic syndrome or steroid-dependent nephrotic syndrome: a multicentre, double-blind, randomised, placebo-controlled trial. Lancet 384:1273-1281. doi: 10.1016/S01406736(14)60541-9 
14. KDIGO (2012) KDIGO clinical practice guideline for glomerulonephritis. Kidney Interrnational Supplements 2:143-153. doi: 10.1038/kisup.2012.13

15. Lombel RM, Gipson DS, Hodson EM (2013) Treatment of steroid-sensitive nephrotic syndrome: new guidelines from KDIGO. Pediatr Nephrol 28:415-426. doi: $10.1007 / \mathrm{s} 00467-012-2310-\mathrm{x}$

16. MacHardy N, Miles PV, Massengill SF, Smoyer WE, Mahan JD, Greenbaum L, Massie S, Yao L, Nagaraj S, Lin JJ, Wigfall D, Trachtman H, Hu Y, Gipson DS (2009) Management patterns of childhood-onset nephrotic syndrome. Pediatr Nephrol 24:2193-2201. doi: $10.1007 / \mathrm{s} 00467-009-1282-\mathrm{y}$

17. Mekahli D, Liutkus A, Ranchin B, Yu A, Bessenay L, Girardin E, Van DammeLombaerts R, Palcoux JB, Cachat F, Lavocat MP, Bourdat-Michel G, Nobili F, Cochat P (2009) Long-term outcome of idiopathic steroid-resistant nephrotic syndrome: a multicenter study. Pediatr Nephrol 24:1525-1532. doi: 10.1007/s00467-009-1138-5

18. Nakanishi K, Iijima K, Ishikura K, Hataya H, Nakazato H, Sasaki S, Honda M, Yoshikawa N (2013) Two-year outcome of the ISKDC regimen and frequent-relapsing risk in children with idiopathic nephrotic syndrome. Clinical journal of the American Society of Nephrology : CJASN 8:756-762. doi: 10.2215/CJN.09010912

19. Nakayama M, Katafuchi R, Yanase T, Ikeda K, Tanaka H, Fujimi S (2002) Steroid responsiveness and frequency of relapse in adult-onset minimal change nephrotic syndrome. American journal of kidney diseases : the official journal of the National Kidney Foundation 39:503-512. doi: 10.1053/ajkd.2002.31400

20. Pasini A, Aceto G, Ammenti A, Ardissino G, Azzolina V, Bettinelli A, Cama E, et al. (2015) Best practice guidelines for idiopathic nephrotic syndrome: recommendations versus reality. Pediatr Nephrol 30:91-101. doi: 10.1007/s00467-014-2903-7

21. Samuel S, Morgan CJ, Bitzan M, Mammen C, Dart AB, Manns BJ, Alexander RT, Erickson RL, Grisaru S, Wade AW, Blydt-Hansen T, Feber J, Arora S, Licht C, Zappitelli M (2013) Substantial practice variation exists in the management of childhood nephrotic syndrome. Pediatr Nephrol 28:2289-2298. doi: 10.1007/s00467-013-2546-0

22. Sellier-Leclerc AL, Baudouin V, Kwon T, Macher MA, Guerin V, Lapillonne H, Deschenes G, Ulinski T (2012) Rituximab in steroid-dependent idiopathic nephrotic syndrome in childhood--follow-up after CD19 recovery. Nephrology, dialysis, transplantation : official publication of the European Dialysis and Transplant Association European Renal Association 27:1083-1089. doi: 10.1093/ndt/gfr405

23. Sellier-Leclerc AL, Belli E, Guerin V, Dorfmuller P, Deschenes G (2013) Fulminant viral myocarditis after rituximab therapy in pediatric nephrotic syndrome. Pediatr Nephrol 28:1875-1879. doi: 10.1007/s00467-013-2485-9

24. Sinha A, Saha A, Kumar M, Sharma S, Afzal K, Mehta A, Kalaivani M, Hari P, Bagga A (2015) Extending initial prednisolone treatment in a randomized control trial from 3 to 6 months did not significantly influence the course of illness in children with steroidsensitive nephrotic syndrome. Kidney international 87:217-224. doi: 10.1038/ki.2014.240

25. Tarshish P, Tobin JN, Bernstein J, Edelmann CM, Jr. (1997) Prognostic significance of the early course of minimal change nephrotic syndrome: report of the International Study of Kidney Disease in Children. Journal of the American Society of Nephrology : JASN 8:769-776. doi:

26. Yoshikawa N, Nakanishi K, Sako M, Oba MS, Mori R, Ota E, Ishikura K, Hataya H, Honda M, Ito S, Shima Y, Kaito H, Nozu K, Nakamura H, Igarashi T, Ohashi Y, Iijima K (2015) A multicenter randomized trial indicates initial prednisolone treatment for childhood nephrotic syndrome for two months is not inferior to six-month treatment. Kidney international 87:225-232. doi: 10.1038/ki.2014.260 
27. Zagury A, Oliveira AL, Montalvao JA, Novaes RH, Sa VM, Moraes CA, Tavares Mde S (2013) Steroid-resistant idiopathic nephrotic syndrome in children: long-term followup and risk factors for end-stage renal disease. Jornal brasileiro de nefrologia : 'orgao oficial de Sociedades Brasileira e Latino-Americana de Nefrologia 35:191-199. doi: $10.5935 / 0101-2800.20130031$ 